\title{
Cultural factors within the united states promote substance use disorders: a helpful perspective for responding to the opioid misuse epidemic
}

\begin{abstract}
Among the many factors that explain society's responses to addiction and substance abuse behaviors, the cultural factors are significant and pervasive. In contrast to the physiological factors related to substances of abuse, such as their pharmacology, dose, potency, frequency, and duration of use, cultural factors are often challenging to identify, much less quantify. Elsewhere the reader can review more comprehensive discussions of cultural factors that are involved in substance use disorders.1-4 As for the individual, the collective denial of cultural factors, particularly problematic ones, is common. Prohibition and legal measures to limit access and availability have been the primary tools used to curb substance abuse. Given the public health implications of ongoing substance abuse and the urgency for finding effective solutions, new and potentially transformational approaches warrant consideration.
\end{abstract}

Volume 4 Issue I - 2017

\section{J Kimber Rotchford}

Olympas Pain and Addiction Services (OPAS), USA

Correspondence: J Kimber Rotchford, Olympas Pain and Addiction Services (OPAS), I I 36 Water Street, Suite 107, Port Townsend,WA 98368, USA, Tel 360-385-4843, Email jkrotchford@gmail.com

Received: March 18, 2017 | Published: October 17, 2017

\section{Introduction}

The subject matter related to cultural factors in medicine, particularly in addiction medicine is vast. Why then discuss these factors ever so briefly? The understanding of cultural factors is valuable. Moreover, as with personal as well as social change, getting into the solutions is the intent. I hope the discussions herein prompt a more robust Public Health response rather than a further reliance on our criminal justice and regulatory approaches. The FDA and the DEA are relatively unique and novel American experiments. Their purported aim is to better assure the safe and effective use of substances, whether pharmaceutical or natural. While the benefits of regulatory oversight have been demonstrated, the unintended and direct consequences of regulatory zeal are apparent. Administrative law and the regulatory apparatus in the United States have been mushrooming for decades and informed observers are legitimately concerned.

The populations of the United States and many other countries around the world are challenged by an acute, public health crisis related to opioid use and abuse. Because opioids have a well-established and important clinical utility in medical care, we cannot simply prohibit or severely limit their use without serious, unintended consequences. Less than one hundred years ago the United States experimented with the prohibition of alcohol. In retrospect, it is widely acknowledged as a failed experiment. Now, the same cultural factors that prompted the prohibition of "intoxicating liquors" by passage of the Eighteenth Amendment to the United States Constitution are still present. In addition to alcohol, which has some social and health benefits, other abused substances have little clinical or social redeeming qualities. The abuse of substances such as cocaine and methamphetamines continues to be a serious problem despite the huge investments and sustained efforts to eliminate abuse through prohibition and government regulations.

The federal government's "War on Drugs" is commonly judged to be a failed social experiment. In countries such as Portugal and Switzerland, where drug abuse and its consequences are more successfully contained, we see a common pattern of active, public health management with harm reduction strategies. In these successful experiments, the public health approaches replace prohibition and criminal-justice responses. Opioids remain valuable medications to treat patients suffering from serious acute and chronic pain as well as those patients suffering from opioid use disorders, i.e., addiction. It is highly doubtful that a continuing emphasis on prohibition and regulatory efforts to manage opioid abuse will have salutary outcomes.

\section{Cultural factors}

Cultural beliefs vis-à- vis addiction and substance abuse blind people to facts, scientific principles, the experience of others, and the wisdom of experts. Preconceived notions commonly dominate facts and scientific reasoning. The simplicity and political appeal of the "Just say no" approach is apparent. This approach is coupled with the often unspoken belief that, if one cannot say "No," then one needs to be punished. Rehabilitative efforts in American jails and prisons are given "lip service." Compared to other developed countries our American legal system is highly adversarial and punitive. The consequences are commonly brutal. This is particularly true when dealing with dysfunctional behavior related to mental illnesses such as substance use disorders. The amount Americans invest in their criminal justice system surpasses that of any other developed country. Are Americans safer? Do they have less crime? Do they have less drug addiction? Are they truly freer with a large percentage of their population in jails and prisons and with the large number of citizens highly indebted to the criminal justice system? In addition to prohibition, punishment, and "Just say no" approaches, what are some of the other cultural factors that promote the high rates of substance abuse in America? What is it about the American culture that has cultivated this epidemic and now produces high morbidity and mortality that arguably surpasses any other culture, aside perhaps from the rates of alcoholism in Russia and Eastern Europe? In the past, when we have tried to slow down one substance use epidemic, it seems like another substance use epidemic raises its head. Often the consequences of subsequent substance use epidemics are even more serious.

In my career, I have seen this happen repeatedly. A good example is the recent attempt in Washington State to stop the epidemic of overdoses stemming from prescription opiates. This intervention, touted as "successful," was accomplished on the back of increased regulatory efforts aimed at physicians. One likely unintended consequence was the number of people dying from heroin overdoses. This increased mortality from heroin overdoses does not even include 
the serious morbidity associated with the suffering, the disabilities, and the mortality rates resulting from poorly managed pain. In Washington State, many patients are frustrated in their struggle to find physicians prepared to adequately address their pain management needs. While the overprescribing of opioids is a real problem, the lack of access to adequate pain management is also a serious and potentially deadly outcome as well.

This lack of access to adequate pain management care was likely hampered by the regulatory processes put into place. As witnessed in the Washington State example, society's inclination to over regulate and criminalize the behavior associated with a disease is significant. It is arguably the most important cultural factor contributing to the high rate of substance abuse. As already mentioned, this pattern of outlawing the use of a substance is consistent with our American penchant toward prohibition. When it comes to dysfunctional and illegal behavior associated with mental health concerns, substance use disorders are a common reason. It is not a coincidence that most inmates in our jails have substance use disorders and other mental health disorders. Indeed, cultural factors related to mental illness and its management should be part of a comprehensive look at cultural factors related to substance use disorders.

Our inclination to blame someone or something is another cultural factor which negatively impacts our approach to substance use disorders. Addicts, criminals, institutions, drug companies, specific substances, physicians, and even lenient prosecutors are blamed. In the airline industry, once blame and shame were replaced by a culture valuing a collective responsibility and a system's approach, accidents were dramatically reduced and airlines achieved significant cost savings. No one can be expected to have a solid grip on all the cultural factors involved. The factors are complex and multifactorial. With that said, our high rates of substance use disorders in the United States are not simply related to genetics or the specifics of our population. This would seem foolish given the "mixing pot," which our American genetic pool represents. Do Americans have less willpower or are they less morally inclined? While never formally studied, I doubt it.

In the resources referenced at the end of the paper, other cultural factors are more thoroughly explored. ${ }^{1-4}$ It is worth noting that recent research has demonstrated that even the language we use to describe mental illness and addiction makes a difference in how sufferers are treated by professionals and by society. ${ }^{5-8}$ Perhaps even the language we use may be a determinant in predicting the incidence or prevalence of substance use disorders? The next few sections on epidemiology and public health are abbreviated but hopefully helpful in justifying a robust public health response to the current opioid use epidemic.

\section{Epidemiology}

Diseases and mental illness are present everywhere. When a disease becomes epidemic or unusually prevalent, cultural and other environmental factors typically play important roles. Epidemiology is the study of factors that promote and sustain epidemics. Common environmental factors associated with infectious disease epidemics, such as weather, flora, fauna, public hygiene, or geography play no significant role in the high rate of addictions in the United States. Cartels and criminals operate on both sides of the country's southern border. They capitalize on the demand for illicit substances of abuse. Nonetheless, to blame our substance abuse epidemic on our geography, that is, because of our proximity to neighbours south of the border, is at best simple minded. Epidemiologists and experts in public health study factors that predict or maintain an epidemic. Many established factors predict the increased risk for substance abuse. These include:

a. The more readily available an abusable substance is, the more likely it is that a certain portion of the population will use and abuse it.

b. The nature of addiction requires repeated exposure to the substance or to a behavior over time. While some people might feel or think they were addicted after the first puff from a cigarette or after the first snort of cocaine, addictive processes require repeated exposure.

c. The easier someone learns complex information or the more natural "reward" one experiences from using a substance, the easier it is for that person to develop a substance use disorder.

d. There are genetic factors and comorbid conditions that contribute to the risk of developing an addiction.

The above risk factors operate in concert with one another. They make sense. They are consistent with facts. And, experts agree. Nonetheless, despite negative evidence, the popular notions that prevail are "Just Say No," have some spine, and make good choices. These beliefs justify society's punitive inclinations when substance abusers make "poor choices." Other predisposing factors commonly evaluated in epidemiology are "vectors." These are, if you like, "carriers of a disease" that act as vectors which increase the likelihood of someone being exposed to the disease. In a malaria epidemic, for example, certain mosquitos, and by extension their habitats, act as vectors for the transmission of the disease. Once exposed for whatever reason to a mosquito (the vector), a person can subsequently come down with malaria.

While the control of vectors for disease prevention is essential, it would be rather foolish to blame, shame, or imprison people (the hosts). Unfortunately, this is commonly how we address those who have been exposed, for whatever the reason, and have contracted a substance use disorder and are suffering the consequences. It is a fact that approximately twenty percent of Vietnam veterans, who were exposed to heroin, came down with the disease. Twenty percent reflects a similar percentage of patients who, because of chronic opioid agonist therapy (COAT) for pain, eventually "contract" an opioid use disorder, i.e., become addicted. Why do we tend to blame and shame the fraction of unfortunates who acquire the disease? No evidence supports the proposition that a lack of willpower or moral integrity protect one from contracting a substance use disorder. On the other hand, there is significant evidence for other risk factors such as genetics, the age at first exposure, past trauma, and others. Epidemiology helps us use reason, statistics, and science coupled with social, financial, legal, and organizational expertise to better manage an epidemic.

\section{Public health}

Public health officials use epidemiology coupled with "soft sciences" to best address an epidemic. To effectively address an epidemic, public health officials search for associated "environmental factors," which promote the vectors associated with a disease, whether the disease is infectious or not. Therefore in regions where malaria exists, authorities attempt to eliminate standing water, whenever possible. This helps assure that the mosquitos cannot effectively breed. When cultural and environmental factors are effectively addressed, 
and, as a result, vectors are curtailed, only then is the disease and an outbreak effectively managed. Any public health official, in addition to addressing the predisposing factors, recognizes the importance to assure effective treatment to those suffering from the disease. In some cases, people carrying the disease need to be quarantined and, while officials commonly have that authority, it is rarely needed.

Those trained in public health also appreciate the need for a coordinated and comprehensive approach when it comes to controlling an epidemic. All members of the healthcare team and important players in the larger community are to be involved. Blaming, shaming, and imprisoning those with a disease do not improve outcomes and arguably make matters worse. While there is an occasional place for law enforcement, it is rarely needed. Based on research and longstanding expertise public health officials are not highly attached to the total eradication of a disease. They recognize such attachment can be counterproductive. Harm reduction approaches commonly work best and can limit the "unintended" and real consequences from overzealous control efforts. As with many clinical and chronic diseases such as diabetes and heart disease, one best manages the diseases and limits their consequences by active management without serious attempts to eradicate the disease.

\section{Discussion}

Given the above discussion, what are possible solutions for substance use epidemics in the United State? One cannot readily legislate cultural factors away. As alluded to above, when it comes to predicting human behavior, facts and reason often do not matter as much as preconceived notions, beliefs, and conditioned responses. It follows that we need to be given opportunities to critically evaluate our beliefs and associated feelings regarding substance misuse and learn new ways to respond. Perhaps it is time for us to deeply grieve and to be open to new ways of believing about and addressing substance misuse? Our collective approach perhaps is to be like that seen for patients who struggle to emerge from the dysfunctional patterns associated with substance use disorders. They are often baffled as to know what to do next. Nothing they try seems to work! Indeed, as commonly observed with substance use disorders, after a brief period of abstinence, the use and dysfunctional patterns can all come back, and often with a vengeance. Would it be helpful for us to entertain, that as communities, we behave like an individual with a substance use disorder?

\section{Cultural transformation}

It does seem that as a culture we are behaving like those who have bona fide substance use disorders. If this analogy holds, our recovery might well need to be part of a cultural transformation. While the term "cultural transformation" might appear extreme, the newcomer to effectively addressing a substance use disorder must confront a sort of personal transformation. The answers often eventually lie, at least behaviourally, in accepting that we are not in control. The paradox is: the more we can accept that we are not in control, the more likely we can start to exhibit some control! For the individual, as well as for our culture, this represents a transformative process. Efforts at more control have been our preferred solution. As with the popular motto "Just say no," despite all the evidence that this approach is ineffective and that threats and scare tactics may make matters worse, many still believe that these outdated behavioral approaches work best. What works best in most cases does not necessarily mean it works best in all the cases. We do need to leave room for exceptions.

\section{Conclusion/Recommendation}

Reasonable options to respond to the opioid abuse crisis have been suggested. ${ }^{9}$ A comprehensive and system evaluation, with clear ways to assure quality outcomes must be planned and utilized. We must stop blaming, shaming, writing more laws, handing out stiffer penalties, filling more prisons, deploying more police and expanding the efforts of regulatory agencies, such as the D.E.A. If the financial incentives for supplying drugs is limited, as we learn from the cases of Al Capone and others, so will the criminal activity. Prevention and effective care for substance use disorders, including opioid use disorders, must be readily available and affordable on demand. This would be like immunizations and other public health interventions aimed at curbing epidemics. Physicians are trained, licensed, and professionally responsible to provide recommendations and prescriptions most likely to help an individual patient. There are already longstanding and established means to promote professional medical care. When regulators and third parties attempt to determine and legislate individual medical care, even with the best of intentions, the unintended consequences are likely significant.

Our cultural inclination toward prohibition is noteworthy. People may believe, if the punishment is severe enough, this resolves the problem. It is hard to argue against this type of belief. As with other belief systems, one can contend that the punishment or intervention was simply not severe or potent enough to have the intended effect! Indeed, it often takes time for established beliefs and conditioned responses to be mitigated by reason and established evidence. We forget that it took multiple generations for people to come to accept that the world is round and not flat. A more robust public health involvement is indicated in addressing the opioid abuse epidemic as with other substance use epidemics. This is the reasonable solution. If we use the principles known to be effective in addressing epidemics, it follows that we can expect better outcomes for an epidemic.

There are significant challenges in making transformational changes, particularly when there are longstanding cultural and financial barriers. The criminal justice system is a major industry within the United States and to compromise it warrants an expectation for significant push back. We must hope for courageous leadership. The biases and prejudices directed toward people with addictive disorders, particularly involving illicit substances, is comparable to those associated with longstanding racial prejudices.

To meet these sorts of challenges, whether personally or as a community, it is often helpful to examine and leverage our values. Our values can be the most important levers we have to effectuate personal as well as cultural changes. Let us value life and a compassion for our neighbours and those who suffer. These are values we hopefully share and can strive to uphold. I also encourage mercy. I find mercy trumps justice in promoting healthy relationships and communities. Mercy means nothing without justice, yet mercy may well be the cornerstone upon which worthwhile lives are built.

Shakespeare reminded us in the Merchant of Venice:

"The quality of mercy is not strain'd

It droppeth as the gentle rain from heaven

Upon the place beneath, it is twice bless'd:

It blesseth them that give, and them that take." 


\section{Acknowledgements}

None.

\section{Conflict of interest}

The author declares no conflict of interest

\section{References}

1. Horvath TA, Misra K, Epner AK, et al. Addiction and Sociological Influences: Culture and Ethnicity. Mental Help Net, USA; 2016.

2. Abbott P, Chase DM. Culture and Substance Abuse: Impact of Culture Affects Approach to Treatment. Psychiatric Times, USA; 2008.

3. Rotchford JK. An Informal Review of Opioid Dependence (Addiction) Associated with Chronic Opioid Analgesic Therapy (COAT) for Chronic Pain. Adiktologie. 2015;15(3).

4. Rotchford JK. Info lays out a background for understanding the curren American opioid epidemic. There is an exploration of the myths surrounding opioid abuse, review of possible cultural influences as well as other risk factors. Arguments for a more robust public health response are provided. 2015
5. Botticelli MP, Hoh HK. Changing the language of addiction. JAMA. 2016;316(13):1361-1362

6. Kelly JF, Westerhoff CM. Does it matter how we refer to individuals with substance-related conditions? A randomized study of two commonly used terms. Int J Drug Policy. 2010;21(3):202-207.

7. Kelly JF, Dow SJ, Weserhoff C. Do our choice of substance-related terms influence perceptions of treatment need? An empirical investigation with two commonly used terms. J Drug Issues. 2010;40(4):805-818.

8. Changing the Language of Addiction. White House Office of the National Drug Control Policy. USA; 2016. p. 1-7.

9. America's 8-Step Program for Opioid Addiction. New York Times Editorial Board, USA; 2017. 